\title{
Use of Anti-Dementia Drugs Reduces the Risk of Potentially Inappropriate Medications: A Secondary Analysis of a Nationwide Survey of Prescribing Pharmacies
}

\author{
Yusuke Suzuki $^{a}$ Mikio Sakakibara $^{\mathrm{b}}$ Nariaki Shiraishic ${ }^{\mathrm{C}}$ Hitoshi Komiya $^{\mathrm{a}}$ \\ Masahiro Akishitad ${ }^{d}$ Masafumi Kuzuya ${ }^{e}$ \\ ${ }^{a}$ Center for Community Liaison and Patient Consultations, Nagoya University Hospital, Nagoya, Japan; b ${ }^{\text {b }}$ gi \\ Pharmacy Co., Ltd., Obu, Japan; 'Department of Rehabilitation, Faculty of Health Sciences, Nihon Fukushi University, \\ Nihon, Japan; dDepartment of Geriatric Medicine, Graduate School of Medicine, The University of Tokyo, Tokyo, \\ Japan; e Department of Community Healthcare and Geriatrics, Nagoya University Graduate School of Medicine, \\ Nagoya, Japan
}

\section{Keywords}

Anti-dementia drugs · Nationwide survey · Potentially inappropriate medications $\cdot$ Prescribing pharmacies

\begin{abstract}
Aims: As the number of older people with dementia increases, safe pharmacotherapy in this population has attracted attention in recent years. The aims of this study were to clarify the prescribing patterns in older patients who were prescribed anti-dementia drugs and to investigate the association of potentially inappropriate medications (PIMs) with the use of anti-dementia drugs. Methods: Adults aged $\geq 65$ years, who were prescribed anti-dementia drugs at 585 pharmacies across Japan $(N=7,953)$, were surveyed. The percentage of prescriptions of anti-dementia drugs and the effect of those prescriptions on PIMs were investigated. Results: Prescriptions of anti-dementia drugs were found in $4.4 \%$ of the entire study population. A multiple logistic regression analysis revealed that the use of anti-dementia drugs reduced the risk of prescribing psychotropic drugs, which represented
\end{abstract}

karger@karger.com www.karger.com/dem

Karger $\stackrel{\text { ' }}{5}$

GOPEN ACCESS
(C) 2020 The Author(s)

Published by S. Karger AG, Basel

This is an Open Access article licensed under the Creative Commons Attribution-NonCommercial-4.0 International License (CC BY-NC) (http://www.karger.com/Services/OpenAccessLicense), applicable to the online version of the article only. Usage and distribution for commercial purposes requires written permission.
PIMs, and that a combination of anti-dementia drugs (e.g., cholineesterase inhibitor with memantine) may reduce the risk of prescribing PIMs compared with monotherapy. Conclusion: The use of anti-dementia drugs was associated with fewer prescriptions of drugs considered as PIMs.

(C) 2020 The Author(s)

Published by S. Karger AG, Basel

\section{Introduction}

Increased attention has been given to prescription safety in routine clinical practice, where a large proportion of patients are of older age and are vulnerable to potentially inappropriate medications (PIMs) relative to younger patients [1]. In our previous study based on a nationwide survey of prescribing pharmacies across Japan, which aimed to clarify prescribing patterns in older patients and factors associated with PIMs, we found that an increased proportion of PIMs in older patients were not due to advanced age per se but to a disproportionately increasing number of prescribed medications, wide- 
ly known as polypharmacy. Regarding the association with the class of drugs, the use of psychotropic medications was found to be largely responsible for PIMs in the sampled population [2], which suggested that reducing the number of medications, in particular deprescribing neuropsychiatric drugs based on careful medication reviews, may reduce the risk of PIMs in older patients; this would then decrease undesirable drug-induced adverse events that have been reported elsewhere $[3,4]$.

Moreover, some conditions are common in old age but are not sufficiently recognised by clinicians, which eventually leads to underuse of the indicated medications [5, 6]. Certain people with dementia (PWD) who are considered underdiagnosed in typical primary practice [7] despite the constant increase in prevalence may be exposed to various health risks stemming from comorbidities [8$10]$ as well as adverse drug reactions, which are presumably induced by potential exposures to neuropsychiatric drugs $[11,12]$ or poor competence in their medication management. According to a survey implemented in 6 Japanese municipalities in 2012, the number of individuals with dementia in Japan was estimated to account for $15.7 \%$ of the entire older population. This figure was much higher than what had been previously estimated [13] and inevitably raised social awareness to address various problems related to dementia care. Many individuals with dementia exhibit behavioural and psychological symptoms of dementia (BPSD). Treatment guidelines for BPSD have long warned clinicians of the potential harms of extended or inappropriate use of antipsychotic medications in older patients. The United States Food and Drug Administration also released a statement, which suggests that both conventional and atypical antipsychotics are associated with an increased risk of mortality in older patients treated for dementia-related psychosis [14]. Other than the potential risk of selecting antipsychotics for the treatment of BPSD, attention must be given to various adverse drug reactions, which may affect the functional prognosis in PWD. Therefore, clarifying the prescribing patterns in older PWD compared with those of non-demented older patients may be of significance in terms of improving prescription safety in growing demented populations.

In this secondary analysis of data from a nationwide survey of prescribing pharmacies in Japan, we aimed to clarify the prescribing patterns of older patients who were prescribed anti-dementia drugs by comparing them with those who were not prescribed those drugs and to investigate the association of PIMs with the use of anti-dementia drugs.

Use of Anti-Dementia Drugs Reduces the Risk of PIMs
Table 1. Number and percentage of older adults prescribed with anti-dementia drugs $(N=7,953)$

\begin{tabular}{lrr}
\hline & Patients & $\begin{array}{l}\text { Proportion, } \\
\%\end{array}$ \\
\hline ChEI monotherapy & & \\
$\quad$ Donepezil & 4,195 & 52.7 \\
$\quad$ Galantamine & 861 & 10.8 \\
$\quad$ Rivastigmine & 668 & 8.4 \\
$\quad$ Subtotal (monotherapy) & 5,724 & 72.0 \\
Memantine monotherapy & 1,173 & 14.7 \\
Combination therapy & 1,056 & 13.3 \\
$\quad$ (ChEI + memantine) & 7,953 & 100.0 \\
\hline Total & & \\
\hline
\end{tabular}

ChEI, cholinesterase inhibitor.

\section{Methods}

Data Source and Acquisitions

In order to avoid sampling bias, the present survey analysed data collected from prescribing pharmacies distributed throughout Japan. Records of all prescriptions issued to patients over 65 years of age between October 1 and October 31, 2014, at 585 dispensing pharmacies across the nation (145 in metropolitan areas, 243 in central Japan, and 197 in western Japan) were collected. The pharmacies receive prescriptions issued for outpatients who visit all types of medical institutions irrespective of clinical settings. Before acquisition of data, written informed consent was obtained from all participants after attending pharmacists provided them with a complete description of the study. Data from each individual, which included age, sex, number, and type of drugs prescribed, were coded before collection to assure anonymity. The types of drugs were classified based on a physiological system and were otherwise classified as "other" in accordance with the drug classification adopted by the STOPP/START criteria [15].

\section{Data Analysis}

Among the entire sampled population, individuals who were prescribed any anti-dementia drugs (donepezil, galantamine, rivastigmine, and memantine) were analysed in the present study. The number of patients who were prescribed each anti-dementia drug and the number of patients with combined medications (any cholinesterase inhibitor [ChEI] with memantine) were counted and the number of total prescriptions was calculated for comparison. In order to examine the involvement of anti-dementia medications in the association between the type of drug and prescriptions for PIMs, patients who were prescribed drugs that affect the central nervous system (CNS drugs) were subcategorised into those who were prescribed any anti-dementia medications and those who were not. To determine the factors associated with the prevalence of PIMs, a univariate analysis followed by a multivariable logistic regression analysis adjusted for age, sex, number of drugs, and class of drugs prescribed as covariates, were performed to calculate odds ratios and 95\% confidence intervals (95\% CIs). Drugs that were included in the list of medications released by the

Dement Geriatr Cogn Disord 2020;49:526-532 
Table 2. Factors associated with PIMs (JGS list): univariate and multivariate analyses $(n=180,673)$

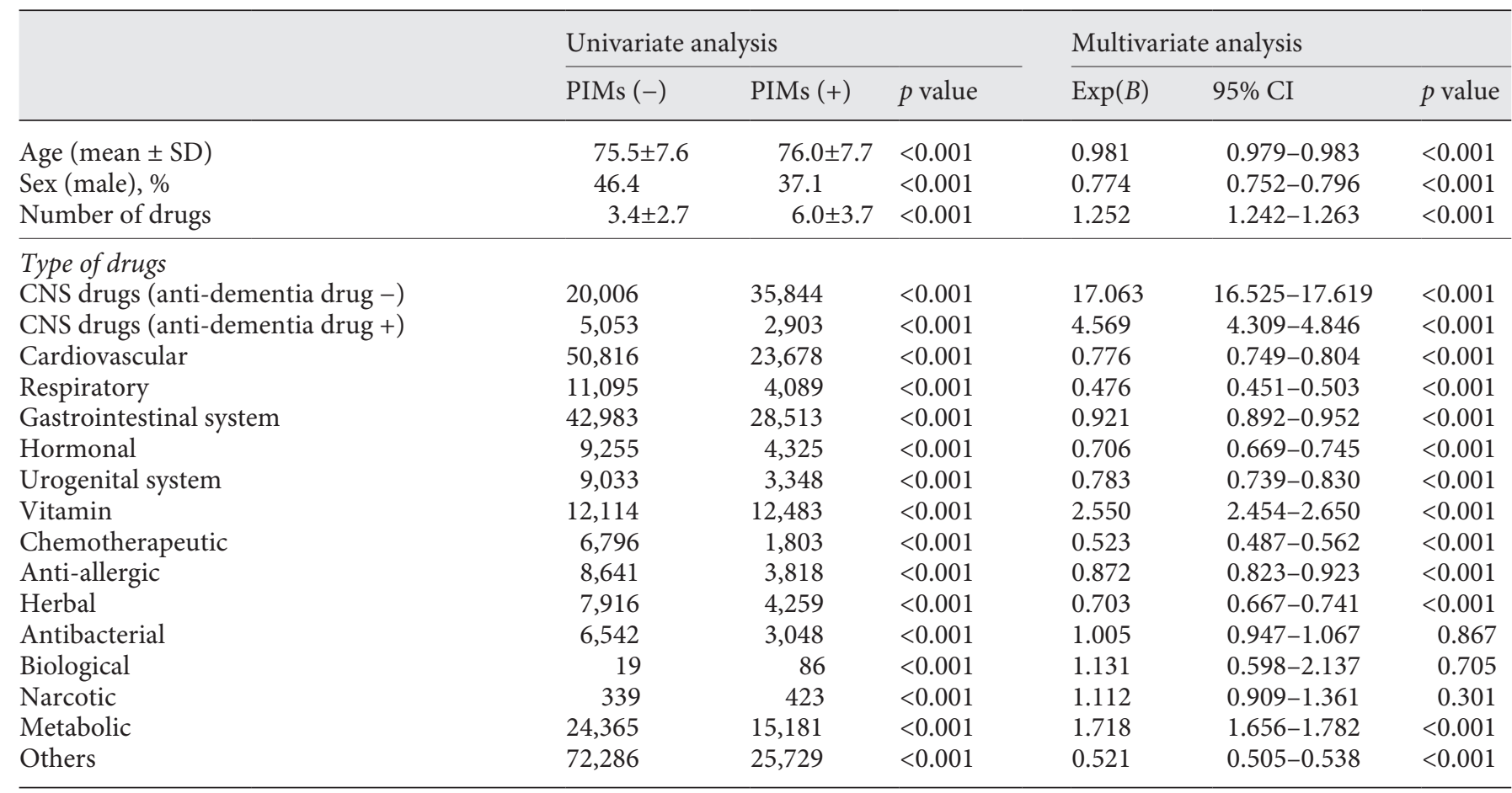

PIM, potentially inappropriate medication; JGS, Japan Geriatrics Society; CI, confidence interval; CNS, central nervous system.

Japan Geriatrics Society (hereafter referred to as the JGS list) were considered equivalent to PIMs in the present study. The JGS list includes drugs that need to be prescribed with special caution based on thorough systematic reviews of previous studies [16].

In order to examine the association of anti-dementia medications with the risk of PIMs, anti-dementia medications were categorised as either a monotherapy, which includes any ChEIs or memantine alone, or a combined therapy, which includes any ChEI combined with memantine. Another univariate analysis, in which anti-dementia medications were the independent variables, was performed followed by a multivariable logistic regression analysis adjusted for age, sex, and number of drugs as covariates. All analyses were performed using a statistical software package (SPSS version 25.0 for Windows; SPSS Inc., Chicago, IL, USA), and $p<$ 0.05 was considered to denote statistical significance.

\section{Results}

Over the study period, 7,953 (4.4\%) out of 180,673 older adults were prescribed anti-dementia drugs. Of those who were prescribed anti-dementia drugs, $72.0 \%(n=$ $5,724)$ were prescribed 1 ChEI only, while $14.7 \%(n=$ 1,173 ) were prescribed memantine only (monotherapy). Both a ChEI and memantine were prescribed in $13.3 \%$ $(n=1,056)$ of older adults prescribed anti-dementia drugs (combination therapy) (Table 1). A multivariate analysis involving the total study population was performed to examine the associations of variables with PIMs and revealed that the number of drugs and sex (female), but not age, were associated with PIMs. Regarding the associations among the types of drugs, CNS drugs, vitamins, and metabolic drugs were significantly associated with PIMs in the multivariate analysis. When the prescriptions for CNS drugs were categorised according to the use of antidementia drugs, those who were prescribed anti-dementia drugs had a much lower risk of PIMs compared with those who were not prescribed those drugs (Table 2). When only subjects who were prescribed anti-dementia drugs were considered, sex (female), number of drugs, and vitamins remained significantly associated with PIMs, while metabolic drugs were negatively associated with PIMs (Table 3). Among those who were prescribed anti-dementia medications, those prescribed monotherapy consisting of memantine alone had an increased risk of PIMs $(\operatorname{Exp}(B): 1.423,95 \%$ CI: $1.239-1.636, p<0.001)$ compared with monotherapy consisting of any ChEI (served as a reference). In contrast, those prescribed a 
Table 3. Factors associated with PIMs (JGS list) in patients prescribed anti-dementia drugs: univariate and multivariate analyses $(n=$ $7,953)$

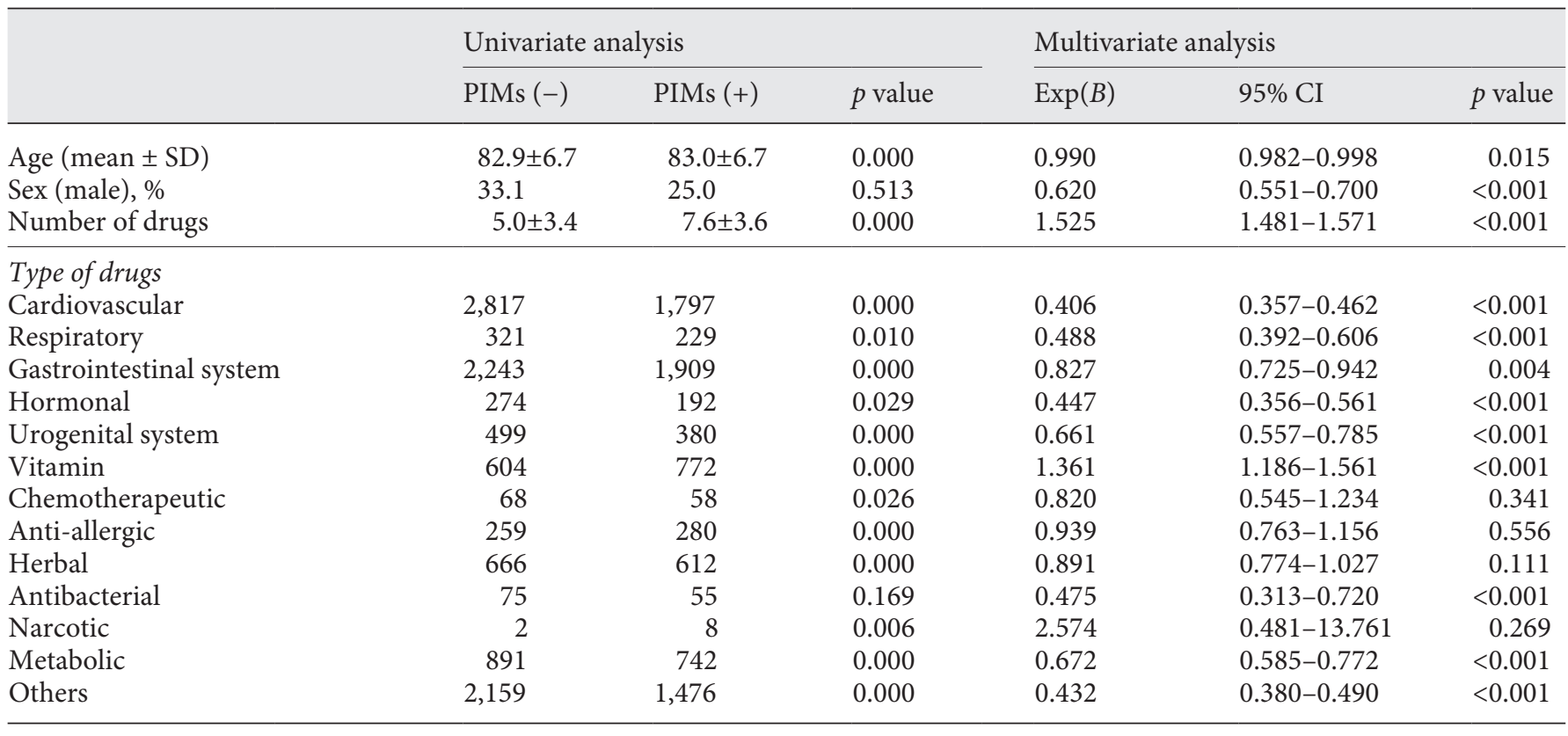

PIM, potentially inappropriate medication; JGS, Japan Geriatrics Society; CI, confidence interval.

Table 4. Factors associated with PIMs (JGS list) by pharmacotherapy of anti-dementia drugs: univariate and multivariate analyses $(n=$ $7,953)$

\begin{tabular}{|c|c|c|c|c|c|c|}
\hline & \multicolumn{3}{|c|}{ Univariate analysis } & \multicolumn{3}{|c|}{ Multivariate analysis } \\
\hline & PIM (-) & $\operatorname{PIM}(+)$ & $p$ value & $\operatorname{Exp}(B)$ & $95 \% \mathrm{CI}$ & $p$ value \\
\hline Age $($ mean $\pm S D)$ & $82.9 \pm 6.7$ & $83.0 \pm 6.7$ & 0.513 & 0.978 & $0.971-0.986$ & $<0.001$ \\
\hline Sex (male), \% & 33.1 & 25.0 & $<0.001$ & 0.578 & $0.516-0.647$ & $<0.001$ \\
\hline Number of drugs (mean \pm SD) & $5.0 \pm 3.4$ & $7.8 \pm 3.6$ & $<0.001$ & 1.254 & $1.236-1.272$ & $<0.001$ \\
\hline ChEI monotherapy (reference) & 3,739 & 1,985 & & 1 & & $<0.001$ \\
\hline Memantine monotherapy & 668 & 505 & $<0.001$ & 1.423 & $1.239-1.636$ & $<0.001$ \\
\hline ChEI + memantine (combination therapy) & 645 & 411 & 0.77 & 0.979 & $0.846-1.131$ & 0.770 \\
\hline
\end{tabular}

PIM, potentially inappropriate medication; JGS, Japan Geriatrics Society; CI, confidence interval; ChEI, cholinesterase inhibitor.

combination therapy had a slightly reduced risk, although the difference was not statistically significant $(\operatorname{Exp}[B]$ : 0.979 , 95\% CI: 0.846-1.131, $p=0.77$ ) (Table 4).

\section{Discussion}

In this secondary analysis of a nationwide survey, we confirmed that the prescription of anti-dementia drugs was disproportionally less common, given the estimat- ed prevalence of older patients diagnosed with dementia of Alzheimer type in the study population. Fewer prescriptions of anti-dementia drugs relative to the expected number of patients who could have clinical indications may simply reflect an underdiagnosis of dementia in general clinical settings, which may lead to undertreatment by relevant medications; alternatively, a poor continuing rate for various reasons $[17,18]$ or concerns for unexpected adverse drug reactions may explain the observed results. Given a previous report 
suggesting a potentially excessive use of anti-dementia drugs in patients above the age of 85 [19], who are not generally included in clinical trials, careful clinical considerations are desired in the application of anti-dementia medications.

Similar to our previous report [2], polypharmacy and female gender were significantly associated with PIMs in older patients when the study population was limited to those who were prescribed anti-dementia drugs. The results also agree with findings from a previous report involving older adults with mild cognitive impairment [20]. Our multivariable analysis also showed that vitamins were the only factor that was positively associated with PIMs. Prescriptions for diabetic drugs (sulfonylureas, biguanides, or thiazolidinediones), which are included in the JGS list, exhibited a positive association with PIMs when all the study populations were included, but such an association disappeared when included individuals were limited to those prescribed anti-dementia medication. A possible association of antidiabetic medications and the risk of dementia, presumably via hypoglycaemic episodes, has also been reported [21, 22], and such a risk is reflected in previously released recommendations/guidelines for diabetic control in older adults [23]. Therefore, a prescriber may be cautious in treating diabetic patients who are considered to have dementia. On the contrary, a significant association between vitamins and PIMs was observed irrespective of prescriptions for anti-dementia drugs, which is intriguing since no vitamins are included in the JGS list. The breakdown of vitamins prescribed showed that vitamin $\mathrm{D}$ and vitamin B complex/multivitamins accounted for the majority (data not shown). The attitude of practitioners towards prescribing drugs such as vitamin B complex/multivitamins, whose long-term clinical efficacies are not well-established [24, 25], may represent awareness of drug safety, which might have affected the association of such drugs with prescriptions of PIMs. Demented patients, who often present BPSD, are considered to have a greater risk of being prescribed CNS drugs such as neuroleptics or anxiolytics, which leads to an elevated risk of PIMs [26]. However, among those who were prescribed CNS drugs, we observed a remarkable reduction in PIM risk in individuals who were prescribed anti-dementia drugs compared with those who were not prescribed those drugs. Several possible reasons for the observed risk reduction may be considered. First, the application of anti-dementia drugs may have prevented the manifestation of BPSD in the study population, which may have, in turn, reduced the prevalence of the use of CNS drugs for symptomatic control of behavioural problems [27, 28]. Second, in PWD, the continuation rate of ChEI prescription has been shown to decline over time with disease progression $[18,29]$, and thus, those who were prescribed anti-dementia drugs might have been in earlier stages of dementia with less necessity for pharmacological treatment of BPSD with CNS drugs potentially categorised as PIMs. Conversely, in case of patients with advanced stage of dementia, prescriptions of ChEIs might have been abstained due to concerns over exacerbations of BPSD and those of neuroleptics, mainly responsible for PIMs, might have been considered instead. Third, contrary to the suggested indifference of prescribers in the treatment of older patients with anti-dementia drugs, as reflected by the conspicuous underuse relative to the estimated size of the patient population, those who dare prescribe anti-dementia drugs may be proactive towards the care of older patients and may possess a higher awareness of safer pharmacotherapy in this fragile population. According to the analysis focussing on anti-dementia drugs, compared with ChEI monotherapy, those who were prescribed memantine had a significantly elevated risk of PIMs, but that risk may be mitigated by the combination of a ChEI with memantine. Other than the expected long-term neuroprotective effects, memantine is often used for symptoms such as irritability and aggressiveness, which are often present in dementia patients [30], and patients who demonstrate such symptoms are likely to be co-prescribed memantine for symptomatic treatment and CNS drugs that fall into the category of PIMs.

A strength of our study is that the survey is believed to represent prescribing patterns of generic healthcare settings regardless of the venue since the data were sampled equally from wide areas across the country. Nonetheless, our study also has some shortcomings. Due to limitations in the available data collection, we could not obtain key information such as past history of illness, current clinical diagnoses, or comorbidities, which may be useful for determining factors associated with PIMs in each individual. The number of PIMs, which would have served to denote the degree of inappropriateness, was also not available. Additionally, important pharmacological information such as dose, duration, or adherence to medication was not included in our analyses. As mentioned above, since clinical information was not available and was therefore not part of the data collection, not all individuals who were prescribed anti-dementia drugs may have met the diagnostic criteria for dementia, and the degree of their dementia symptoms is 
therefore unknown. Since the data in the present study were collected for 1 month in 2014, prescribing patterns and the prevalence of PIMs in the study population may have changed since then. Collection of more recent and specific data to investigate the factors related to PIMs and how those factors affect clinical consequences would be warranted in our future investigations. In conclusion, our secondary analysis of a nationwide survey revealed that the use of anti-dementia drugs or the combination of available drugs (e.g., ChEI with memantine) was associated with fewer prescribing of PIMs, in particular, CNS drugs.

\section{Acknowledgements}

The authors wish to thank all the patients who agreed to participate in this study and the pharmacists working for Sugi Pharmacy, where the data were collected.

\section{Statement of Ethics}

This study was implemented in compliance with the Declaration of Helsinki, and the study protocol was approved by the Ethical Committee of the Nagoya University Graduate School of Medicine (No. 2014-0195). All the participants have given written informed consent prior to data collection.

\section{Conflict of Interest Statement}

Y.S. received lecture fees from Daiichi Sankyo and MSD. M.A. received lecture fees from Daiichi Sankyo, Mitsubishi-Tanabe Pharma, MSD, Sumitomo Dainippon Pharma, and Takeda Pharmaceutical, and research funding from Astellas Pharma, Bayer HealthCare, Boehringer Ingelheim, Chugai Pharmaceutical, Daiichi Sankyo, Eli Lilly Japan, Eisai, Kracie Pharma, Mitsubishi-Tanabe Pharma, MSD, Novartis Japan, Ono Pharmaceutical, Sanofi, Takeda Pharmaceutical, Teijin Pharma, and Tsumura. M.K. received lecture fees from Eisai, Ono Pharmaceutical, Daiichi Sankyo, Otsuka Pharmaceutical, and Tsumura, and research funding from Daiichi Sankyo, Takeda Pharmaceutical, Eisai, Pfizer, Sumitomo Dainippon Pharma, Astellas Pharma, and Ono Pharmaceutical. All other authors have no conflicts of interest to declare. The funder had no role in the design, methods, data collections, analysis, or preparation of the paper.

\section{Funding Sources}

This study was supported by a Grant-in Aid from the Ministry of Health, Labour and Welfare of the Japanese government (Longevity Sciences Grant No. 25150501).

\section{Author Contributions}

Y.S. conceptualized methodology and made original draft preparation and wrote final manuscript. M.S. conceptualized design of the study and curated data. N.S. and H.K. conducted statistical analysis. M.A. obtained grant funding and helped to design the study. M.K. provided advice on overall protocol of the study.

\section{References}

1 Corsonello A, Pedone C, Lattanzio F, Lucchetti M, Garasto S, Di Muzio M, et al. Potentially inappropriate medications and functional decline in elderly hospitalized patients. J Am Geriatr Soc. 2009;57(6):1007-14.

2 Suzuki Y, Sakakibara M, Shiraishi N, Hirose T, Akishita M, Kuzuya M. Prescription of potentially inappropriate medications to older adults. A nationwide survey at dispensing pharmacies in Japan. Arch Gerontol Geriatr. 2018 Jul-Aug; $77: 8-12$.

3 Nishtala PS, Fois RA, McLachlan AJ, Bell JS, Kelly PJ, Chen TF. Anticholinergic activity of commonly prescribed medications and neuropsychiatric adverse events in older people. J Clin Pharmacol. 2009;49(10):1176-84.

4 Gray MP, Dziuba G, Quach K, Wong A, Smithburger PL, Seybert AL, et al. Assessing adverse drug reactions from psychotropic medications reported to the U.S. food and drug administration in older adults. Am J Geriatr Psychiatry. 2019;27(2):181-5.

5 Wright RM, Sloane R, Pieper CF, Ruby-Scelsi C, Twersky J, Schmader KE, et al. Underuse of indicated medications among physically frail older US veterans at the time of hospital discharge: results of a cross-sectional analysis of data from the Geriatric Evaluation and Management Drug study. Am J Geriatr Pharmacother. 2009;7(5):271-80.

6 Cherubini A, Ruggiero C, Dell'Aquila G, Eusebi P, Gasperini B, Zengarini E, et al. Underrecognition and undertreatment of dementia in Italian nursing homes. J Am Med Dir Assoc. 2012;13(8):759-13.

7 Connolly A, Gaehl E, Martin H, Morris J, Purandare N. Underdiagnosis of dementia in primary care: variations in the observed prevalence and comparisons to the expected prevalence. Aging Ment Health. 2011;15(8):97884.

8 Chen TB, Yiao SY, Sun Y, Lee HJ, Yang SC, Chiu MJ, et al. Comorbidity and dementia: a nationwide survey in Taiwan. PLoS One. 2017;12(4):e0175475.

9 Poblador-Plou B, Calderón-Larrañaga A, Marta-Moreno J, Hancco-Saavedra J, SicrasMainar A, Soljak M, et al. Comorbidity of de- mentia: a cross-sectional study of primary care older patients. BMC Psychiatry. 2014;14:84.

10 Callahan CM, Schubert CC. Dementia: the complexities and comorbidity in dementia. Nat Rev Neurol. 2014;10(4):184-6.

11 Delgado J, Bowman K, Clare L. Potentially inappropriate prescribing in dementia: a stateof-the-art review since 2007. BMJ Open. 2020; 10(1): $\mathrm{e} 029172$.

12 Kanagaratnam L, Mahmoudi R, Novella JL, Jolly D, Dramé M, Trenque T. Adverse drug reactions in elderly subjects hospitalized in a specialized dementia management unit. Drugs Aging. 2014;31(10):769-76.

13 Shadlen MF, Larson EB, Yukawa M. The epidemiology of Alzheimer's disease and vascular dementia in Japanese and African-American populations: the search for etiological clues. Neurobiol Aging. 2000;21(2):171-81.

14 Gill SS, Bronskill SE, Normand SL, Anderson GM, Sykora K, Lam K, et al. Antipsychotic drug use and mortality in older adults with dementia. Ann Intern Med. 2007;146(11): 775-86.
Use of Anti-Dementia Drugs Reduces the Risk of PIMs
Dement Geriatr Cogn Disord 2020;49:526-532 DOI: $10.1159 / 000512043$ 
15 Gallagher P, Ryan C, Byrne S, Kennedy J, O'Mahony D. STOPP (screening tool of older Person's prescriptions) and START (screening tool to alert doctors to right treatment). Consensus validation. Int J Clin Pharmacol Ther. 2008;46(2):72-83.

16 Kojima T, Mizukami K, Tomita N, Arai H, Ohrui EM, Takeya Y, et al. Screening tool for older Persons' appropriate prescriptions for Japanese: report of the Japan Geriatrics Society Working Group on "guidelines for medical treatment and its safety in the elderly". Geriatr Gerontol Int. 2016;16(9): 983-1001.

17 Maclagan LC, Bronskill SE, Guan J, Campitelli MA, Herrmann N, Lapane KL, et al. Predictors of cholinesterase discontinuation during the first year after nursing home admission. J Am Med Dir Assoc. 2018;19(11): 959-66.

18 Umegaki H, Itoh A, Suzuki Y, Nabeshima T. Discontinuation of donepezil for the treatment of Alzheimer's disease in geriatric practice. Int Psychogeriatr. 2008;20(4):800-6.

19 Okumura Y, Sakata N. Antidementia drug use in Japan: bridging the research-to-practice gap. Int J Geriatr Psychiatry. 2018 May 20; 33(9):1286-7.

20 Weston AL, Weinstein AM, Barton C, Yaffe K. Potentially inappropriate medication use in older adults with mild cognitive impairment. J Gerontol A Biol Sci Med Sci. 2010; 65(3):318-21.

21 Cheng C, Lin CH, Tsai YW, Tsai CJ, Chou $\mathrm{PH}$, Lan TH. Type 2 diabetes and antidiabetic medications in relation to dementia diagnosis. J Gerontol A Biol Sci Med Sci. 2014; 69(10):1299-305

22 Whitmer RA, Karter AJ, Yaffe K, Quesenberry CP Jr, Selby JV. Hypoglycemic episodes and risk of dementia in older patients with type 2 diabetes mellitus. JAMA. 2009;301(15): $1565-72$.

23 GMellitus in Older People. A brief statement of key principles of modern day management including the assessment of frailty. a natl collaborative stakeholder initiative. Diabet Med. 2018;35(7):838-45.

24 Jenkins DJA, Spence JD, Giovannucci EL, Kim YI, Josse R, Vieth R, et al. Supplemental vitamins and minerals for CVD prevention and treatment. J Am Coll Cardiol. 2018; 71(22):2570-84.

25 Butler M, Nelson VA, Davila H, Ratner E, Fink HA, Hemmy LS, et al. Over-the-counter supplement interventions to prevent cognitive decline, mild cognitive impairment, and clinical Alzheimer-type dementia: a systematic review. Ann Intern Med. 2018;168(1):5262.
26 Wucherer D, Eichler T, Hertel J, Kilimann I, Richter S, Michalowsky B, et al. Potentially inappropriate medication in community-dwelling primary care patients who were screened positive for dementia. J Alzheimers Dis. 2017; 55(2):691-701.

27 Daiello LA, Ott BR, Lapane KL, Reinert SE, Machan JT, Dore DD. Effect of discontinuing cholinesterase inhibitor therapy on behavioral and mood symptoms in nursing home patients with dementia. Am J Geriatr Pharmacother. 2009;7(2):74-83.

28 Cummings J, Lai TJ, Hemrungrojn S, Mohandas E, Yun Kim S, Nair G, et al. Role of donepezil in the management of neuropsychiatric symptoms in Alzheimer's disease and dementia with Lewy bodies. CNS Neurosci Ther. 2016;22(3):159-66.

29 Johnell K, Religa D, Eriksdotter M. Differences in drug therapy between dementia disorders in the Swedish dementia registry: a nationwide study of over 7,000 patients. Dement Geriatr Cogn Disord. 2013;35(5-6):239-48.

30 Martinez C, Jones RW, Rietbrock S. Trends in the prevalence of antipsychotic drug use among patients with Alzheimer's disease and other dementias including those treated with antidementia drugs in the community in the UK: a cohort study. BMJ Open. 2013;3: e002080. 